\title{
Remote detection of single emitters via optical waveguides
}

\author{
Patrick Then, ${ }^{1}$ Gary Razinskas, ${ }^{1}$ Thorsten Feichtner, ${ }^{1, *}$ Philippe Haas,,${ }^{2, \dagger}$ Andreas Wild,,${ }^{2, \ddagger}$ Nicola Bellini,, 3, \\ Roberto Osellame, ${ }^{3}$ Giulio Cerullo, ${ }^{3}$ and Bert Hecht ${ }^{1, \|}$ \\ ${ }^{1}$ Nano-Optics \& Biophotonics Group, Department of Experimental Physics 5, Röntgen Research Center for Complex Materials (RCCM), \\ Physics Institute, University of Würzburg, Am Hubland, D-97074 Würzburg, Germany \\ ${ }^{2}$ Augenzentrum Bern, Länggassstrasse 18, CH-3012 Bern, Switzerland \\ ${ }^{3}$ Istituto di Fotonica e Nanotecnologie - CNR and Dipartimento di Fisica - Politecnico di Milano, Piazza L. Da Vinci 32, 20133 Milano, Italy
}

(Received 27 February 2014; published 1 May 2014)

\begin{abstract}
The integration of lab-on-a-chip technologies with single-molecule detection techniques may enable new applications in analytical chemistry, biotechnology, and medicine. We describe a method based on the reciprocity theorem of electromagnetic theory to determine and optimize the detection efficiency of photons emitted by single quantum emitters through truncated dielectric waveguides of arbitrary shape positioned in their proximity. We demonstrate experimentally that detection of single quantum emitters via such waveguides is possible, confirming the predicted behavior of the detection efficiency. Our findings blaze the trail towards efficient lensless single-emitter detection compatible with large-scale optofluidic integration.
\end{abstract}

DOI: 10.1103/PhysRevA.89.053801

PACS number(s): 42.25.Bs, 42.82.Bq, 07.07.Df

\section{INTRODUCTION}

The detection of single fluorescent emitters represents the ultimate limit of analytical chemistry. It evades the necessity to investigate ensembles and allows probing both static and dynamic inhomogeneities, thereby providing a tool to study, e.g., the time evolution or steady states of biochemical or biophysical interactions at a molecular level [1,2]. In recent years the integration of single-emitter detection within labon-a-chip architectures has attracted significant interest, since it would allow the combination of the ultimate sensitivity of single-emitter detection, handling of ultrasmall analyte volumes and parallel processing. However, the detection of single emitters is usually thought to require bulky microscope objectives with high numerical aperture to achieve both a small detection volume and a large solid angle for photon capture $[3,4]$. An alternative route to achieve single-emitter detection is the use of near-field optical probes or more generally, "optical nanoantennas", that at the same time facilitate a strong local excitation of emitters and a boosted collection of photons via enhancement of the local optical density of states [5,6]. However, when applying nanoantennas the necessity of detection optics is not removed and in addition, the available detection volume becomes very small, well below $\lambda^{3}$, putting constraints on the usable analyte concentrations [7]. As a consequence, integration of single-emitter detection in

\footnotetext{
*Currently with Max-Planck-Institute for the Science of Light, Günther-Scharowski-Str. 1, Bldg. 24, 91058 Erlangen.

${ }^{\dagger}$ Currently with Clinic St. Anna, Lucerne, Hirslandengruppe, Department of Internal Medicine, St. Anna-Strasse 32, 6006 Lucerne, Switzerland.

${ }^{\ddagger}$ Currently with Augenklinik Hohmad, Hohmad Privatklinik Thun, CH-3600 Thun, Switzerland.

${ }^{\S}$ Currently with SUPA, School of Physics, and Astronomy, University of St. Andrews, North Haugh, KY16 9SS St. Andrews, Scotland, UK.

"To whom correspondence should be addressed: hecht@physik. uni-wuerzburg.de
}

lab-on-a-chip systems has so far proven difficult [8,9]. To realize a fully integrated single-emitter detection system, a combination of dielectric optical waveguides with microfluidics seems promising [10,11]. However, truncated dielectric waveguides are commonly considered to have too low photon collection efficiency and a too low signal-to-noise ratio (SNR) to allow for direct lens-free single-molecule detection. Nevertheless, recent experiments suggest that single-molecule detection in aqueous solution via dielectric waveguides is possible [12,13]. In the present paper we confirm by theoretical considerations, numerical simulations, and experiments that single-emitter detection via dielectric waveguides is indeed possible. We provide a method based on the reciprocity theorem of electromagnetic theory to predict the relevant parameters of the resulting optical system, i.e., the overall detection efficiency and the detection volume, for any type of single-mode waveguide. As a relevant example, we consider a cleaved single-mode optical fiber and demonstrate experimentally the remote detection of single fluorescent colloidal semiconductor quantum dots by placing the cleaved fiber end in their close proximity. Our results confirm the predicted spatial dependence of the detection efficiency. Besides demonstrating remote detection of single emitters using optical waveguides, our work provides the basis for straightforward design and analysis of the optical performance of arbitrary waveguides integrated into microfluidic chips, thereby paving the road to optimized designs and a more widespread application of optofluidic lab-on-a-chip architectures.

\section{THEORY}

We consider a radiating single emitter (SE) — a dye molecule, quantum dot, or any other quantum emitter-close to the end facet of a single-mode waveguide $(W)$ - an on-chip waveguide or an optical fiber-supporting a single guided mode with fields $\boldsymbol{E}_{1}$ and $\boldsymbol{H}_{1}$ [see Fig. 1(a)]. The SE is described as a radiating point dipole situated at $\boldsymbol{r}_{0}$ with dipole moment $\boldsymbol{p}$ that radiates corresponding fields $\boldsymbol{E}_{S E}$ and $\boldsymbol{H}_{S E}$ [14]. To determine the overall detection efficiency of the system, an 

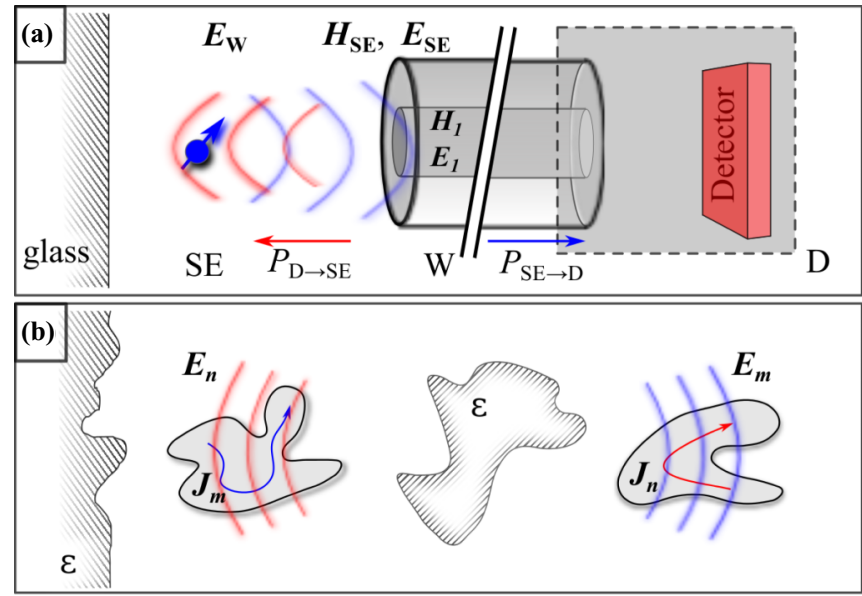

FIG. 1. (Color online) General experimental setting: (a) a single emitter (SE) is placed next to the facet of a lossless single-mode waveguide $(W)$, whose mode is described by fields $\boldsymbol{H}_{1}$ and $\boldsymbol{E}_{1}$. The far end of the fiber combined with the connected detection assembly is treated as detector $(D)$, receiving all power $P_{S E \rightarrow D}$ transmitted through $W$ via the fields $\boldsymbol{H}_{S E}$ and $\boldsymbol{E}_{S E}$. In order to make use of reciprocity, the detector is assumed to emit light as an ideal sender, exciting the SE with the field $\boldsymbol{E}_{W}$ transporting the power $P_{D \rightarrow S E}$. The lower panel (b) sketches a general setting for which reciprocity can be formulated: two source currents $\boldsymbol{J}_{m / n}$ originating in volumes $V_{m / n}$ result in respective far fields $\boldsymbol{E}_{m / n}$, also containing fields reflected and diffracted by the environment described by $\epsilon$.

important figure of merit is the fraction of power coupled from the radiating SE to the waveguide, described by the in-coupling efficiency $\eta\left(\boldsymbol{r}_{0}, \boldsymbol{p}\right)$, a quantity that is usually determined by mode matching [15]:

$\eta=\frac{1}{2 P_{0}} \operatorname{Re}\left(\frac{\int_{A} \boldsymbol{E}_{1} \times \boldsymbol{H}_{S E}^{*} \cdot \hat{z} d A \cdot \int_{A} \boldsymbol{E}_{S E} \times \boldsymbol{H}_{1}^{*} \cdot \hat{z} d A}{\int_{A} \boldsymbol{E}_{1} \times \boldsymbol{H}_{1}^{*} \cdot \hat{z} d A}\right)$.

Here $A$ denotes the area of the waveguide end face, $\hat{z}$ the unit vector parallel to the waveguide, and $P_{0}$ the SE's overall radiated power. For a brief summary of the mode-matching method, see Appendix A. In a general single-molecule detection experiment in complex environments and with diffusing SEs, the determination of $\eta$ for multiple SE positions and orientations requires a new dipolar field calculation or simulation for every configuration to determine the fields $\boldsymbol{E}_{S E}$ and $\boldsymbol{H}_{S E}$ at $A$. We will now show that $\eta$ can be determined for arbitrary locations and orientations of a SE relative to the waveguide with a single field simulation, even for a complex environment. The key to this simplification is the application of the reciprocity theorem that can be used once the electric field $\boldsymbol{E}_{W}$, emitted by the waveguide end face when fed with the mode fields, is known. The Lorentz formulation of electromagnetic reciprocity [16],

$$
\int \boldsymbol{E}_{m} \cdot \boldsymbol{J}_{n} d V_{n}=\int \boldsymbol{E}_{n} \cdot \boldsymbol{J}_{m} d V_{m}
$$

connects two electrical currents $\boldsymbol{J}_{m / n}$ and their respective radiated electric fields $\boldsymbol{E}_{m / n}$ in two finite volumes $V_{m / n}$, separated by an arbitrary environment consisting of particles and/or half-spaces described by linear dielectric constants $\epsilon$ [see Fig. 1(b)]. Considering the definition of the electrical power, we can write

$$
P_{e l}=\int \boldsymbol{E} \cdot \boldsymbol{J} d V
$$

Equation (2) shows that when the tasks of the particles as sender and receiver are exchanged (time reversal symmetry), the transmitted power remains the same:

$$
P_{e l, m \rightarrow n}=P_{e l, n \rightarrow m} .
$$

We now identify particle $M$ with the SE. It is situated near the end of a lossless single-mode fiber $W$ whose far end is directly connected to a photon detector [(Fig. 1(a)]. For simplicity, particle $N$ subsumes the combination $D$ of the detection device assembly and the connected fiber, assuming all power in the fiber mode will be detected. In the reciprocal case, $D$ therefore is a perfect sending device coupling all power into the fiber mode $\boldsymbol{E}_{1}$, leading to the field $\boldsymbol{E}_{W}$ emitted from the fiber facet and exciting the SE. To calculate the deposited electrical power, one has to insert the source current of a point dipole $\boldsymbol{J}_{S E}=i \omega \boldsymbol{p} \delta\left(\boldsymbol{r}-\boldsymbol{r}_{0}\right)$ into (3). We find

$$
\begin{aligned}
P_{S E \rightarrow D} & =\int \boldsymbol{E}_{W} \cdot \boldsymbol{J}_{S E} d V \\
& =\int-i \omega \boldsymbol{p} \cdot \boldsymbol{E}_{W} \delta\left(\boldsymbol{r}-\boldsymbol{r}_{0}\right) d V \\
& =-i \omega \boldsymbol{p} \cdot \boldsymbol{E}_{W}\left(\boldsymbol{r}_{0}\right)
\end{aligned}
$$

Inserting (5) in Eq. (2) and writing the dipole moment for an excited single emitter as $\boldsymbol{p}=\overline{\overline{\boldsymbol{\alpha}}} \cdot \boldsymbol{E}\left(\boldsymbol{r}_{0}\right)$ [17] using the SE's polarizability tensor $\overline{\bar{\alpha}}$ yields the final result:

$$
P_{S E \rightarrow D}=-i \omega \overline{\overline{\boldsymbol{\alpha}}} \cdot \boldsymbol{E}_{W}^{2}\left(\boldsymbol{r}_{0}\right)=P_{D \rightarrow S E} .
$$

This implies that once the vector field $\boldsymbol{E}_{W}$ emitted from the fiber facet is known for a given environment, which can be determined by a single calculation or simulation, also the power of a point dipole coupled into the fiber mode is known for all its possible positions and orientations. Using (6), we can now write $\eta$ as

$$
\eta=P_{S E \rightarrow D} / P_{0}
$$

\section{SIMULATIONS}

To verify the reciprocity-based derivation of the in-coupling efficiency (7), we crosscheck it versus numerical modematching results (1). We therefore calculate (i) the electric field $\boldsymbol{E}_{W}$ that is emitted by the fiber facet and (ii) the electric and magnetic field distributions of a point dipole close to the fiber facet for different dipole positions and orientations with identical geometry by means of finite-difference-time-domain (FDTD) simulations (FDTD Solutions, version 8.0.0, Lumerical Solutions, Inc., Vancouver, Canada). The simulation setup is shown in Fig. 2(a). We model a single-mode fiber by a cylindrical core of dielectric material with index $n=1.4580$ and a diameter of $3.5 \mu \mathrm{m}$ that is surrounded by a cladding of index $n=1.4530$. Figure 2(b) shows the cross section of the near-field intensity distribution of the fundamental fiber mode $(\lambda=800 \mathrm{~nm})$, obtained using a finite-difference-frequencydomain (FDFD) algorithm [18] (MODE Solutions, version 5.0.4, Lumerical Solutions, Inc., Vancouver, Canada). The 


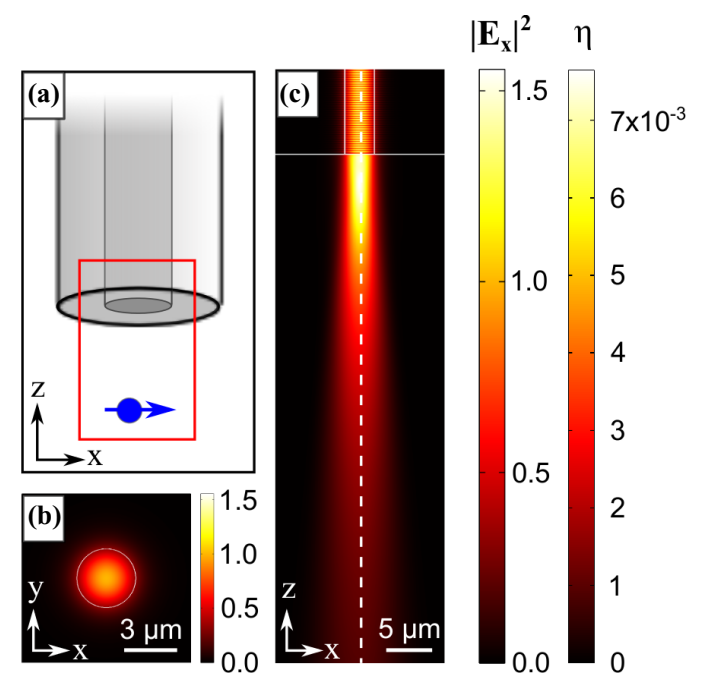

FIG. 2. (Color online) (a) Geometry used in simulations. A single-mode fiber sits at variable distance $z$ above a point dipole. The red box denotes the actual simulation volume, as pictured in (c). (b) FDFD simulation of the fundamental fiber mode profile $(\lambda=800 \mathrm{~nm})$ inside the fiber. (c) $x$ component of the normalized electric field intensity $\left|\boldsymbol{E}_{W}\right|^{2}$ of the out-coupled fundamental fiber mode [longitudinal section including the optical axis (white dashed line) of (a)]. The identical plot can be used to show the position dependence of a SE's in-coupling efficiency $\eta$ when it is oriented along the $x$ axis. The two different scales differ only by a constant factor $\beta$.

single emitter is approximated by a point dipole source with dipole moment parallel to the fiber facet and an emission spectrum modeled as a Gaussian with a center wavelength of $800 \mathrm{~nm}$ and spectral width of $60 \mathrm{~nm}$. Figure 2(c) shows a longitudinal section of the field intensity at a free-fiber facet normalized to the maximum of the fundamental mode propagating within the fiber. As the beam exits the fiber its onaxis intensity first decreases and then increases again to more than 1.5 times the value compared to the fiber-mode maximum before finally decaying towards zero. This peculiar behavior reminds one of a focusing effect, however, it can be readily explained by considering the Bessel-shaped field distribution inside the fiber [19] which results in a very short-ranged Bessel beam directly after the fiber facet exhibiting typical intensity oscillations [20,21]. After a distance of $\approx 5 \mu \mathrm{m}$, the Bessel beam breaks down and the beam starts to diverge, gradually approaching the behavior of a Gaussian beam with a divergence compatible with the fiber's numerical aperture (NA $=0.12$ ).

We now have all the ingredients to perform the aforementioned crosscheck. We find a linear relation between the efficiency $\eta$ of SE power coupled into the waveguide mode and the square of the absolute value of the projection of the fiber-mode field component at the position $\boldsymbol{r}_{0}$ onto the SE's dipole moment unit vector $\hat{\boldsymbol{p}}$ :

$$
\left|\boldsymbol{E}_{W}\left(\boldsymbol{r}_{0}\right) \cdot \hat{\boldsymbol{p}}\right|^{2}=\beta \cdot \eta .
$$

This allows us to calculate the in-coupling efficiency of SE emission into the fiber mode using the intensity distribution caused by mode excitation [see Fig. 2(c), right scale]. For the simulated geometry, evaluating several SE positions along the optical axis, we obtain the scaling factor $\beta=196.2$, with a standard deviation $\sigma_{\beta}=0.5$, which is due to slight spatial variations of $\beta$ caused by numerical artefacts. Using the scaling factor, we calculate a maximum in-coupling efficiency of $\eta=0.79 \%$, corresponding to the SE positioned in the intensity maximum of the out-coupled field. To afford a direct comparison with the setup used in the experiments, we also simulated the intensity distribution and in-coupling efficiency in the presence of a substrate at varying distances (as also depicted in Fig. 1), supporting a layer that contains the single emitters. In these simulations, the dipole source is held in a 200 -nm-thick polymethylmethacrylate (PMMA) layer $(n=1.48431)$ [22] on top of a semi-infinite glass substrate $(n=1.45334$; comparable to the material used in the experiments) [23]. It turns out that the presence of the substrate does not significantly alter the overall shape of the emitted fiber mode but merely leads to shallow interference undulations between the fiber and substrate (see Fig. 6), as well as to a reduced field strength in the substrate due to reflection at the interface (see Appendix B).

\section{EXPERIMENTAL}

\section{A. Materials and methods}

In order to experimentally verify the predicted spatial dependence of the in-coupling efficiency, a carefully cleaned microscope cover slip (Menzel cover glass no. 1.5, thickness $0.17 \mathrm{~mm}$ ) is spincoated with a 100-pM solution of QDot800 ITK carboxyl quantum dots (QDs) (Invitrogen, Life Technologies) and a subsequent 200-nm layer of PMMA. Excitation of the quantum dot fluorescence is provided by an asymmetric dark-field scheme employing an off-axis, needlelike beam that is created by placing a small aperture (diameter $1.5 \mathrm{~mm}$ ) in the beam path of a coherent white-light source $(\lambda=530 \mathrm{~nm}$, SuperK Power with SpectraK AOTF, NKT Photonics) and displacing the lens laterally to the beam axis. The beam is thus focused at an incidence angle close to the critical angle of total internal reflection, which exceeds the numerical aperture of the fiber. This configuration provides efficient excitation of quantum dots while keeping the background in the detection path very low. As the focusing lens we use a microscope objective (1.30 NA, PL Fluotar $100 x$, Leica), resulting in a diffraction-limited spot size of $\approx 1 \mu \mathrm{m} \times 2.5 \mu \mathrm{m}$. For imaging, the sample is scanned in the $x-y$ plane using a piezo stage (P-527.3CD, E-712, PI Physik Instrumente). To collect the fluorescence, a cleaved single-mode optical fiber (FS-SN-3224, Thorlabs) is situated perpendicular to the surface at a variable distance $z$ ranging from about 2 to $50 \mu \mathrm{m}$, adjusted by a piezo positioner (PIFOC, PI Physik Instrumente). To perform a distance calibration, the mechanical contact between fiber and sample is detected by imaging the sample with a CCD camera and observing a sudden defocusing of the sample upon approach of the fiber. Quantum dot fluorescence entering the fiber is outcoupled at the far end and focused onto a single-photon-counting avalanche photodiode (APD) (SPCM-AQR-13, Perkin-Elmer) after passing a bandpass filter (XF-3307, Omega Optical) to remove remaining excitation light. For a schematic drawing of the experimental setup, see Fig. 3. 


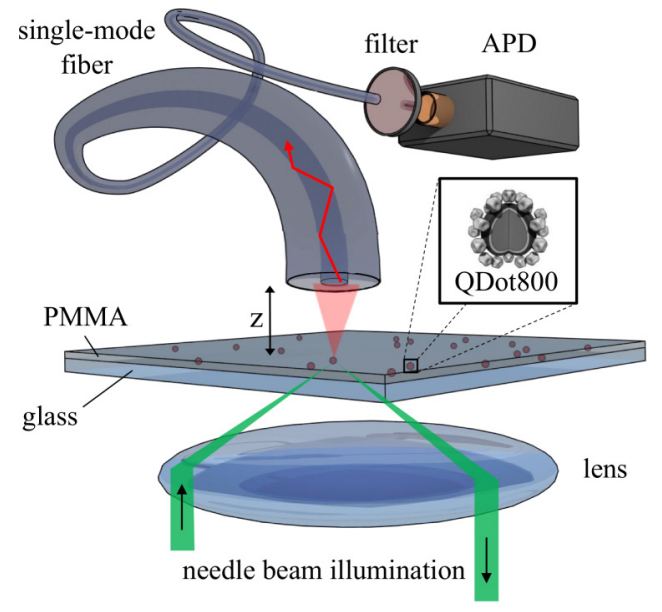

FIG. 3. (Color online) Experimental setup. A single-mode fiber is situated above a microscope cover slip coated with QDot800 (red spheres, inset) in a 200-nm-thick PMMA layer. On the other end of the fiber, the quantum dot fluorescence is delivered to an APD through a filter.

The analysis of recorded scanning images is performed by fitting a two-dimensional (2D) elliptical Gaussian to the recorded spots. Since the fiber gathers light from a sample area comparable to its core diameter, the size and shape of the recorded spots is determined by the illumination point-spreadfunction (PSF) that can therefore be considered constant. After selecting fluorescence spots with a small number of blinking events and fitting the 2D Gaussian, the resulting amplitude is used as a measure for the quantum dot emission coupled into the fiber.

\section{B. Results and discussion}

Figure 4(a) shows three representative images obtained by raster scanning the sample and recording the fluorescence signal at each pixel. Spots of fluorescence emission originating from quantum dots are clearly visible, with a signal-to-noise ratio of up to $\sim 15$. To determine the distance dependence of the in-coupling efficiency into the fiber, we recorded raster scans of the same sample region for a range of sample-fiber distances between 2 and $50 \mu \mathrm{m}$. As the total detected number of photons of each individual QD will depend on the orientation of the QD's emission plane [17] relative to the orientation of the fiber's optical axis, each individual QD spot will exhibit a different maximum amplitude. Therefore, each single-QD distance dependence was normalized to its maximum emission intensity. The experimental data are depicted in Fig. 4(b), together with the normalized analytical and simulation results of the intensity of the out-coupled fiber mode along the optical axis.

Note that several factors add to the relatively large spread we observe in the experimental data. The main contributions are due to fitting uncertainties, caused mainly by the blinking of the QD800, and distance-dependent intensity oscillations due to the presence of the interface and some uncertainty in the absolute fiber-sample distance (cf. Appendix B). Overall, the observed distance dependence of the in-coupling efficiency is in good agreement with our predictions. The detected (a)

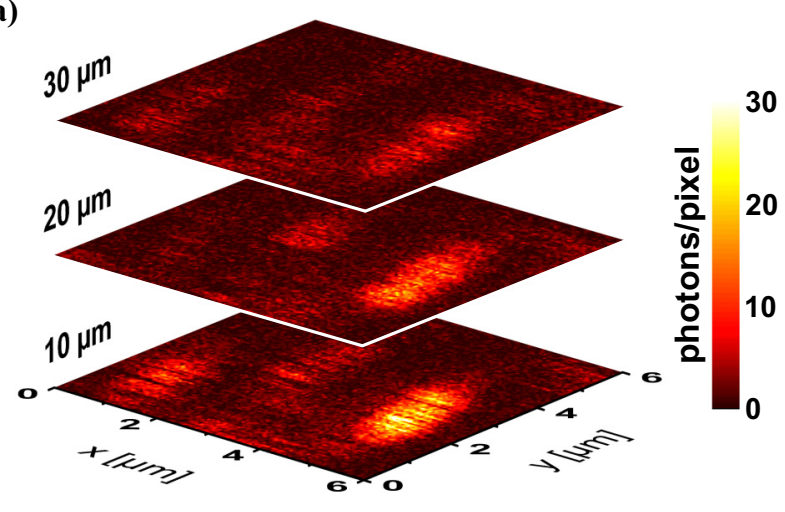

(b)

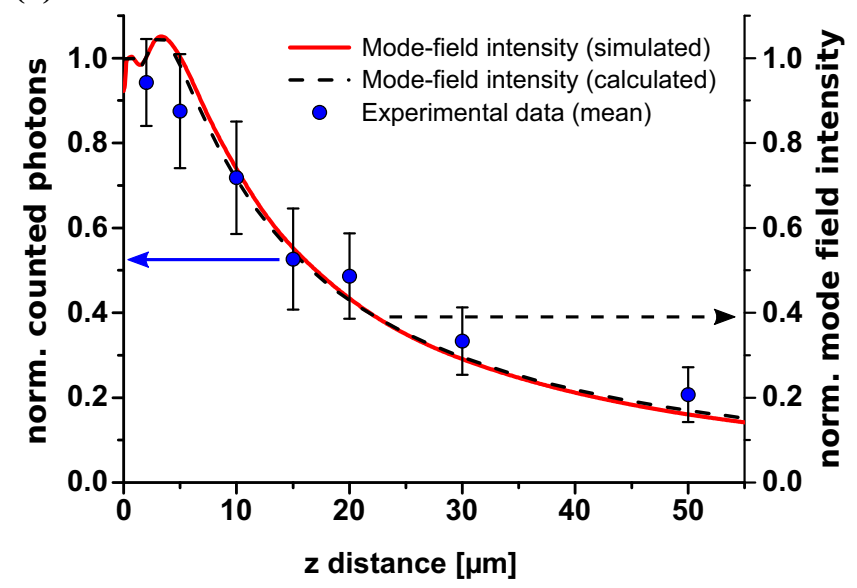

FIG. 4. (Color online) (a) Fluorescence images of a sample area $(6 \times 6 \mu \mathrm{m}, 120 \times 120$ pixels, $3 \mathrm{~ms} /$ pixel $)$ containing the signatures of three single quantum dots recorded at fiber distances of $10 \mu \mathrm{m}, 20 \mu \mathrm{m}$, and $30 \mu \mathrm{m}$ from the PMMA surface. The spots differ in amplitude and exhibit "dark" lines due to blinking. The elongated shape of the spots is a result of the illumination conditions. (b) Normalized photon count from 28 emission spots of QDot800 (left axis). The blue dots indicate the mean value. The red line is showing the simulated-mode field intensity resulting from a cut along the optical axis in Fig. 2(c) (right axis). The dashed black line indicates the analytically calculated-mode field intensity along the optical axis obtained by assumption of a Bessel mode outcoupled at the fiber end-facet (see Appendix C).

quantum dot fluorescence signal is decaying with an increasing gap between the fiber and sample, decreasing to half of the maximum signal at a distance of $\sim 20 \mu \mathrm{m}$. We conclude that in a microfluidic chip, to ensure efficient single-molecule detection via an integrated waveguide with comparable NA, analyte molecules have to pass the waveguide facet within a distance of $20 \mu \mathrm{m}$ or less. The maximum distance to sustain effective single-molecule detection will depend on the characteristic field distribution of the waveguide and the background noise and thus the SNR of the measurement. For an application in a lab-on-a-chip device, the same waveguide can be used for both excitation and detection. Due to the large illuminated liquid volume and possible scattering processes within the waveguide, background noise can become significant, demanding short waveguides and high 


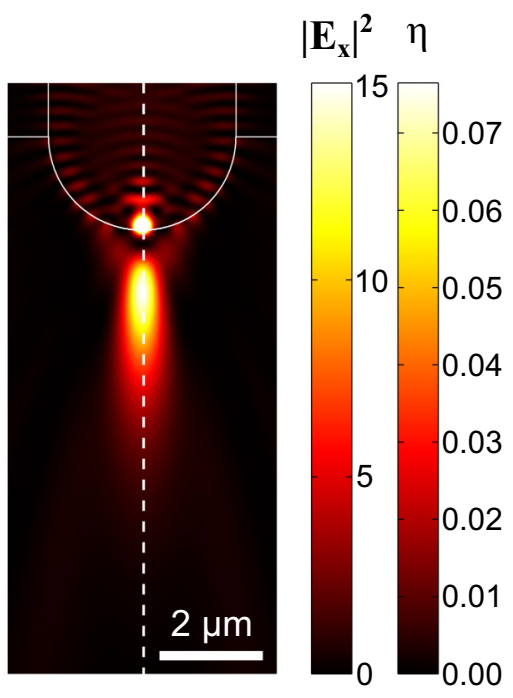

FIG. 5. (Color online) Simulated intensity distribution of a single-mode fiber with a hemispherical lens placed on top of the core. The out-coupled field is concentrated in a small volume, resulting in much higher local intensity and detection efficiency, respectively.

manufacturing quality to maintain a suitable SNR. In an alternative geometry that avoids such problems, two waveguides, one for excitation and one for detection oriented perpendicular to each other, can be used. In both cases the intensity distribution of the out-coupled fields will affect both detection and excitation, which depend on the relative position of the analyte molecule with respect to the waveguides. In comparison to detection schemes employing standard microscope objectives, the overall detection efficiency of waveguide-based systems is low. A typical objective with a numerical aperture of 0.85 will result in a maximum collection efficiency of almost $0.3(30 \%)$ [24], if the molecule's dipole moment is oriented perpendicular to the optical axis. However, it should be noted that the presence of an interface between molecule and objective will limit the maximum collection angle due to total internal reflection at the interface, reducing the efficiency (e.g., $17 \%$ in the presence of a glass cover slip). Nevertheless, we have seen that despite the reduced collection efficiency, single-molecule detection with waveguides can be achieved if background contributions are carefully controlled. The methods presented here can be used to tailor detection schemes through manipulation of the out-coupled field by microlenses or plasmonic structures. As an example, we investigate an optical fiber terminated by a hemispherical lens positioned on top of the guiding core. The resulting field distribution is shown in Fig. 5. This modification yields a maximum field strength and detection efficiency that are 1 order of magnitude larger than those achievable with a simple cleaved fiber. The price to pay for the increased fields is a smaller detection volume, which might be desirable, e.g., for single-molecule experiments at higher concentrations.

\section{CONCLUSIONS}

We have presented a theoretical and numerical method to assess the detection efficiency of arbitrary dielectric waveguides in single-molecule detection experiments. We predict the spatial dependence of the detection efficiency for a single-mode waveguide. We further confirm the predicted behavior experimentally by detecting single semiconductor quantum dots at varying distances along the optical axis from the end facet of an optical fiber. The methods presented here can be employed to optimize arbitrary waveguides for a more efficient, optimized detection, paving the way to compact and fully integrated optofluidic lab-on-a-chip setups.

\section{ACKNOWLEDGMENT}

The authors gratefully acknowledge generous financial support from the LHW Foundation.

\section{APPENDIX A: DERIVATION OF MODE-MATCHING FORMALISM}

We derive the expression for the in-coupling efficiency $\eta\left(\boldsymbol{r}_{0}, \boldsymbol{p}\right)$, as defined in Eq. (1), following [15]. In general, any arbitrary field distribution within a waveguide can be decomposed into the complete basis of waveguide modes, which include a finite number of guided (bound) modes and a continuum of radiating modes. Applied to the present case, the input fields $\boldsymbol{E}_{S E}$ and $\boldsymbol{H}_{S E}$, generated by a radiating SE inside the optical fiber, can thus be written as

$$
\begin{gathered}
\boldsymbol{E}_{S E}(\boldsymbol{r})=\sum_{i} a_{i} \boldsymbol{E}_{i}(\boldsymbol{r})+\boldsymbol{E}_{\mathrm{rad}}(\boldsymbol{r}), \\
\boldsymbol{H}_{S E}(\boldsymbol{r})=\sum_{i} b_{i} \boldsymbol{H}_{i}(\boldsymbol{r})+\boldsymbol{H}_{\mathrm{rad}}(\boldsymbol{r}),
\end{gathered}
$$

where $\boldsymbol{E}_{i}$ and $\boldsymbol{H}_{i}$ are the fields of the $i$ th guided mode, and $\boldsymbol{E}_{\text {rad }}$ and $\boldsymbol{H}_{\text {rad }}$ represent the radiating modes. Using the fiber modes' orthogonality,

$$
\frac{\int_{A} \boldsymbol{E}_{i} \times \boldsymbol{H}_{j}^{*} \cdot \hat{z} d A}{\int_{A} \boldsymbol{E}_{i} \times \boldsymbol{H}_{i}^{*} \cdot \hat{z} d A}=\delta_{i j}
$$

the modal amplitudes $a_{i}$ and $b_{i}$ are defined as

$$
a_{i}=\frac{\int_{A} \boldsymbol{E}_{S E} \times \boldsymbol{H}_{i}^{*} \cdot \hat{z} d A}{\int_{A} \boldsymbol{E}_{i} \times \boldsymbol{H}_{i}^{*} \cdot \hat{z} d A}, \quad b_{i}^{*}=\frac{\int_{A} \boldsymbol{E}_{i} \times \boldsymbol{H}_{S E}^{*} \cdot \hat{z} d A}{\int_{A} \boldsymbol{E}_{i} \times \boldsymbol{H}_{i}^{*} \cdot \hat{z} d A} .
$$

Using the definition of the time-averaged Poynting vector, the total power propagating inside the fiber is then given by

$$
\begin{aligned}
P & =\frac{1}{2} \operatorname{Re}\left(\int_{A} \boldsymbol{E}_{S E} \times \boldsymbol{H}_{S E}^{*} \cdot \hat{z} d A\right) \\
& =\frac{1}{2} \sum_{i} \operatorname{Re}\left(a_{i} b_{i}^{*} \int_{A} \boldsymbol{E}_{i} \times \boldsymbol{H}_{i}^{*} \cdot \hat{z} d A\right),
\end{aligned}
$$

and therefore the fraction of power propagating in the $i$ th mode is

$$
\begin{aligned}
\frac{P_{i}}{P_{0}} & =\frac{1}{2 P_{0}} \operatorname{Re}\left(a_{i} b_{i}^{*} \int_{A} \boldsymbol{E}_{i} \times \boldsymbol{H}_{i}^{*} \cdot \hat{z} d A\right) \\
& =\frac{1}{2 P_{0}} \operatorname{Re}\left(\frac{\int_{A} \boldsymbol{E}_{i} \times \boldsymbol{H}_{S E}^{*} \cdot \hat{z} d A \cdot \int_{A} \boldsymbol{E}_{S E} \times \boldsymbol{H}_{i}^{*} \cdot \hat{z} d A}{\int_{A} \boldsymbol{E}_{i} \times \boldsymbol{H}_{i}^{*} \cdot \hat{z} d A}\right) .
\end{aligned}
$$

For the single guided fiber mode investigated in this study, $i=1$, thus leading to Eq. (1). 


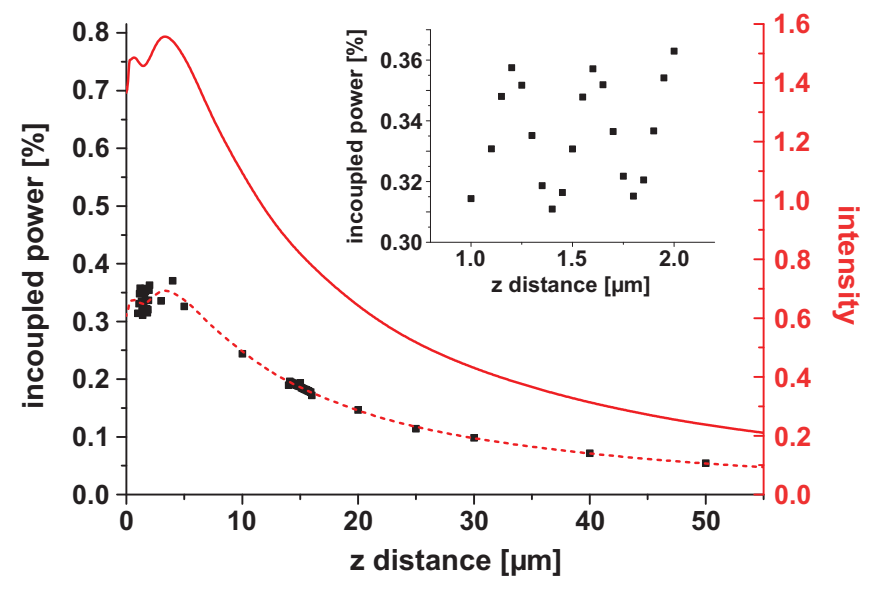

FIG. 6. (Color online) Simulated SE in-coupling efficiency for various distances between fiber and SE. The black dots represent the in-coupled power of a SE embedded in a PMMA layer on top of a glass substrate at various distances to the fiber. The red solid line shows the in-coupled power for the case of a free fiber, obtained by reciprocity. The red dashed line includes the expected reflection at the air-PMMA interface. The scaling of both axes of ordinates is linked via reciprocity. The inset is a magnification of the region around $1.5 \mu \mathrm{m}$ distance.

\section{APPENDIX B: SIMULATIONS OF THE EXPERIMENTAL GEOMETRY}

Since the single emitters studied in the experimental part of this work are embedded in a PMMA layer on top of a glass cover-slip, it is expected that reflections at the airPMMA surface will influence the field distribution between the fiber end and the sample. We simulated the experimental geometry for different fiber-sample distances. The results are shown in Fig. 6 (black dots). The decay we observed without an additional surface is now superimposed by a weak oscillation caused by Fabry-Pérot interferences between the reflecting fiber and sample surfaces, showing a period of half the wavelength. These interference oscillations increase in amplitude for small distances, as shown in the inset of Fig. 6. As a reference, the in-coupled power for the free-fiber case (i.e., no substrate), obtained by reciprocity, is shown in
Fig. 6 (red solid line). While the shape of the curve fits nicely the overall trend of the distance dependence we observed for the previously described geometry, it appears to be shifted to smaller values. This deviation can be explained by reflections at the air-PMMA interface, which lead to lower intensity at the SE position. Owing to reciprocity, the power in-coupled to the fiber mode changes accordingly, when compared to the case of a free fiber. Taking this into account, one can get almost perfect agreement between the free-fiber case and our exact, more complicated geometry (Fig. 6, red dashed line). Additionally, the oscillatory behavior is contributing to the relatively large spread we see in the experimental data. The PIFOC piezo scanner used for stepwise adjusting of the fiber-sample distance provides nanometer accuracy; however, the absolute distance, and therefore the zero point on our $z$ axis, cannot be determined with similar accuracy. Since the uncertainty in fiber-sample distance is on the order of a quarter wavelength, an additional uncertainty about the modulation amplitude of the intensity distribution (about 10\%) in the gap has to be considered.

\section{APPENDIX C: ANALYTICAL CALCULATION OF THE OUT-COUPLED FIBER MODE}

The analytical calculation of the field distribution emitted from the fiber facet has been performed by linking the known field distribution $E(r, 0)$, in our case the field distribution of the fiber mode $\Psi(r, 0)$ at the fiber end, with the field $E(r, Z)$ in a plane $Z$ through the Huygens-Fresnel diffraction integral $[21,25]$. The resulting intensity distribution along the optical axis ( $r=0)$, as shown in Fig. 4(b), is

$$
I(0, z)=\left(\frac{k}{z}\right)^{2}\left|\int_{0}^{a} \rho \Psi(\rho) \exp \left(\frac{i k \rho^{2}}{2 z}\right) d \rho\right|^{2},
$$

where $k$ is the wave number, $z$ the distance from the fiber end facet, and $\Psi$ the propagating fiber mode, consisting of Bessel functions of the first kind $J_{0}(x)$ (in the core) and a modified Bessel function $K_{0}(x)$ (cladding), as determined according to well-known procedures [19]. The integration variable $\rho$ denotes the radial distance, where the upper limit has been chosen such that $K_{0}(x)$ has decayed sufficiently.
[1] Ch. Zander, J. Enderlein, and R. Keller, Single Molecule Detection in Solution Methods and Applications (John Wiley \& Sons, Berlin, 2002).

[2] J. Hohlbein, K. Gryte, M. Heilemann, and A. Kapanidis, Phys. Biol. 7, 031001 (2010).

[3] S. White, H. Li, R. Marsh, J. Piper, N. Leonczek, N. Nicolaou, A. Bain, L. Ying, and D. Klenerman, J. Am. Chem. Soc. 128, 11423 (2006).

[4] A. A. Deniz, S. Mukhopadhyay, and E. A. Lemke, J. R. Soc., Interface 5, 15 (2008).

[5] N. Hosaka and T. Saiki, J. Microsc. (Paris) 202, 362 (2001).

[6] J. N. Farahani, D. W. Pohl, H.-J. Eisler, and B. Hecht, Phys. Rev. Lett. 95, 017402 (2005).
[7] M. J. Levene, Science 299, 682 (2003).

[8] H. Li, L. Ying, J. Green, S. Balasubramanian, and D. Klenerman, Anal. Chem. 75, 1664 (2003).

[9] M. H. Horrocks, H. Li, J. Shim, R. T. Ranasinghe, R. W. Clarke, W. T. S. Huck, C. Abell, and D. Klenerman, Anal. Chem. 84, 179 (2011).

[10] D. Yin, E. Lunt, M. Rudenko, D. Deamer, A. Hawkins, and H. Schmidt, Lab Chip 7, 1171 (2007).

[11] R. M. Vazquez, R. Osellame, D. Nolli, C. Dongre, H. van den Vlekkert, R. Ramponi, M. Pollnau, and G. Cerullo, Lab Chip 9, 91 (2008).

[12] P. Haas, P. Then, A. Wild, W. Grange, S. Zorman, M. Hegner, M. Calame, U. Aebi, J. Flammer, and B. Hecht, Anal. Chem. 82, 6299 (2010). 
[13] P. Ghenuche, H. Rigneault, and J. Wenger, Opt. Express 20, 28379 (2012).

[14] V. Klimov, M. Ducloy, and V. S. Letokhov, Quantum Electron. 31, 569 (2001).

[15] A. W. Snyder, Optical Waveguide Theory, Number 190 in Science paperbacks (Chapman and Hall, London, 1983).

[16] K. Lee, Principles of Antenna Theory (Wiley, New York, 1984).

[17] L. Novotny and B. Hecht, Principles of Nano-Optics (Cambridge University Press, Cambridge, UK, 2012).

[18] Z. Zhu and T. Brown, Opt. Express 10, 853 (2002).

[19] A. K. Ghatak, An Introduction to Fiber Optics (Cambridge University Press, Cambridge, UK, 1998).

[20] J. Durnin, J. Opt. Soc. Am. A 4, 651 (1987).
[21] Z. Jiang, Q. Lu, and Z. Liu, Appl. Opt. 34, 7183 (1995).

[22] S. Kasarova, N. Sultanova, C. Ivanov, and I. Nikolov, Opt. Mater. 29, 1481 (2007).

[23] E. Palik, Handbook of Optical Constants of Solids, Volumes I, II, and III: Subject Index and Contributor Index (Elsevier Science \& Technology, New York, 1985).

[24] T. Plakhotnik, W. E. Moerner, V. Palm, and Urs P. Wild, Opt. Commun. 114, 83 (1995).

[25] Max Born, Principles of Optics: Electromagnetic Theory of Propagation, Interference and Diffraction of Light, 7th expanded ed. (Cambridge University Press, Cambridge, UK, 1999). 